\section{Anmerkungen}

(1) Vgl. Héritier, A. (Hrsg.): Policy-Analyse. Kritiik und Neu-orientierung, Politische Vierteliahresschrift, Sonderheft 24, Opladen 1993;

Prittwitz, V. v.: Politikanalyse, Opladen 1994;

R. Rist: Policy Evaluation. Linking Theory to Practice, Cheltenham 1994;

Rossi, P. H./H. Freeman, E.: Evaluation. A Systematic Approach, Newbury Park, London, New Delhi 1993 (5. Aufl.);

Fischer, F.: Evaluating Public Policy, Chicago 1995.

(2) Vgl. Jänicke, M. (Hrsg.): Umweltpolitik der Industrieländer. Berlin 1996.

(3) Vgl. Jänicke, M., Weidner, H. (eds.): Successful Environmental Policy. A Critical Evaluation of 24 Cases.

Berlin 1995;

Conrad, J.: Successful Environmental Management in European Companies, FFU-report 96-3, Berlin 1996.

(4) Vgl. Jänicke, M. (Hrsg.) a.a.0. 1996; und Mayntz, R.: Zur Einleitung: Probleme der Theoriebildung in der Implementationsforschung, in: Dies. (Hrsg.): Implementation politischer Programme, Opladen 1983.

(5) Luhmann, N.: Ökologische Kommunikation, Opladen 1986.

(6) Mayntz, R. a.a.0. 1983.

(7) Willke, H.: Entzauberung des Staates Überlegungen zu einer sozietalen Steuerungstheorie, Königstein/Ts 1983.

(8) Sabatier, P. A.: Top-down and Bottom-up Approaches to Implementation Research, in: Hill, M. (Ed.): The Policy Process: A Reader, London 1993.

(9) M.Jänicke, H.Mönch, M.Binder, et al.: Umweltentlastung durch industriellen Strukturwandel? Berlin 1993.

(10) Vgl. hierzu Jänicke, M.: Umweltinnovationen aus

Sicht der Policy-Analyse: vom instrumentellen zum strategi-

schen Ansatz der Umweltpolitik. FFU-rep. 97-3,

Berlin 1997.

(11) Vgl. zu entsprechenden Studien am Beispiel der

Reduktion von Gefahrstoffen: Jacob, K., Jänicke, M.: Ökologische Innovationen in der chemischen Industrie: Umweltentlastung ohne Staat? Eine Untersuchung und Kommentierung zu 182 Gefahrenstoffen. FFU-rep 97-4,

Berlin 1997.

(12) OECD: Environmental Taxes and Green Tax Reform, Paris 1997;

European Environment Agency (EEA): Environmental Taxes. Implementation and Environmental Effectiveness, Copenhagen 1996.

\section{Der Autor}

Prof. Dr. Martin Jänicke ist Prolessor für Politikwissenschaft und Leiter der Forschungsstelle fïr Umweltpolitik an der FU Berlin (FFU)

Kontakı: Martin Jänicke, FFU

Schwendener Straße 53, 14195 Berlin

Tel. $++49 /(0) 30 / 8385098$

Eine transdisziplinäre Gestaltungsaufgabe der Politikberatung

\title{
Institutionen für eine nachhaltige Gesellschaft
}

\author{
Sozialwissenschaftliche Schlïsselkonzepte wie dasjenige der "Institution" \\ eröffnen ebenfalls den Zugang zu einer transdisziplinären Wissensgenerierung. \\ Dabei ist die instifutionelle Perspektive von hoher theoretischer und prakti- \\ scher Relevanz in der akfuellen politischen Debatte über die Umsetzung des \\ Konzeptes der Nachhaltigkeit.
}

Von U. Schneidewind, J. Minsch, T. Schulz, J. Wüst, H. P. Meister, P. Feindt, J. Tscheulin

$\mathrm{D}$ ie Diskussion über eine Politik der Nachhaltigkeit läuft heute in vielen Feldern in eine ,Ziel-Instrumenten-Falle": Weithin besteht die Vorstellung, daß in einem ersten Schritt konkrete ökonomische, ökologische und soziale Nachhaltigkeitsziele zu definieren und sie in einem zweiten Schritt mit konkreten Instrumenten umzusetzen seien. Diese auf den ersten Blick plausible Vorstellung abstrahiert jedoch völlig von den konkreten Formen der Entscheidungsfindung in der Gesellschaft sowie den Motivationen der beteiligten Akteure. Die Ergebnisse sind ernüchternd: Die politische Diskussion über Nachhaltigkeit verfängt sich in blockierten Zieldiskussionen und gleichzeitig bleiben Gestaltungspotentiale auf zahlreichen gesellschaftlichen Ebenen ungenutzt. Eine transdisziplinär angelegte Institutionenanalyse kann hier wichtige Impulse für eine nachhaltigkeitsorientierte Politikberatung (1) geben.

\section{- Renaissance der Institutionenperspektive}

Die Auseinandersetzung mit Institutionen spielt in der Politologie, der Soziologie und den Wirtschaftswissenschafen von je her eine wichtige Rolle - in der Regel bezogen auf die spezifischen Fragestellungen der Disziplin. Insbesondere die Volkswirtschaftslehre hat mit der Neuen Institutionenökonomik eine Antwort auf zahlreiche Erklärungsdefizite enger neoklassischer Ansätze entwickelt. Die Etablierung des institutionellen Zweigs der VWL fand nicht zuletzt ihren Ausdruck in den Nobelpreisverleihungen an Ronald Coase und Douglas C. North. Die Endogenisierung von
Institutionen ist dabei eines der zentralen Anliegen der Neuen Instititutionenökonomik (2). Bezogen auf Ihre Anwendungsgebiete ist die Neue Institutionenökonomik interdisziplinär angelegt: Sie beschränkt ihre Analyse nicht nur auf marktliche Strukturen, sondern läßt sich ebenso auf politische Strukturen anwenden. Sie kann als Ausdruck eines ,NeoInstitutionalismus" auch in den anderen Gesellschaftswissenschaften betrachtet werden. Dieser Neo-Institutionalismus repräsentiert eine Art ,Wiederentdeckung" der institutionellen Ebene sozialen Handelns und versucht unter Rückgriff auf Disziplinen wie Politologie, Soziologie, Volkswirtschaftslehre, Rechtswissenschaft, Anthropologie und Philosophie einen interdisziplinär begründeten Mittelweg zwischen akteurszentrierten und funktionalistischen Erklärungsmodellen gesellschaftlicher Prozesse zu entwickeln.

Doch ist die Institutionenanalyse heute nicht nur theoretisch wieder ,en vogue“. Die theoretische öffnung hin $\mathrm{zu}$ Institutionen wird begleitet von einer intensiven Diskussion über Institutionen in der heutigen Gesellschaft. Dies bezieht sich nicht nur - wie in der Einleitung angedeutet - auf die Ergänzung der Zieldiskussion in der Nachhaltigkeitsdebatte um die institutionellen Voraussetzungen einer nachhaltigen Gesellschaft, sondern immer mehr auf die Frage, wie Politik heute insgesamt zu organisieren ist. Zunehmende staatliche Steuerungsdefizite oder Politikverdrossenheit auf der einen Seite, gleichzeitig neue selbstorganisierte Formen der gesellschaftlichen Problemlösung (Branchenselbstverpflichtungen, lokales Engagement etc.) auf der anderen Seite verlangen nach neuen institutionellen Gestaltungsoptionen. 


\section{Schlüsselfragen}

Drei Fragenkomplexe bestimmen dabei die aktuelle Institutionenforschung und -beratung: 1. Sind Institutionen überhaupt gestaltbar oder sind sie vielmehr von ,außen“ vorgegebene und sich unabhängig entwickelnde Rahmenbedingungen?

2. Wer sind die Akteure einer Institutionengestaltung? Welche Rolle spielt der Staat bei der Institutionengestaltung heute und in Zukunft?

3. Nach welchen (normativen) Gestaltungsprinzipien soll eine Institutionengestaltung erfolgen?

Für die Beantwortung aller dieser Fragen bedarf es interdisziplinärer Lösungskompetenz:

1. Die Frage, ob Institutionen Gestaltungsobjekt oder Rahmenbedingung sind, hat die Volkswirtschaftslehre lange modelltheoretisch gelöst, indem sie institutionelle Rahmenbedingungen als exogen gesetzt annahm und das Handeln von rationalen Akteuren in einem gegebenen Institutionenset betrachtete. Mit der Neuen Institutionenökonomik, der evolutorischen Ökonomik, aber auch der u.a. spieltheoretisch inspirierten Theorie internationaler Verhandlungen und Verträge wurden Institutionen selbst zum Erklärungsobjekt. Ob sich die jeweiligen Institutionen als gestaltbar erweisen, hängt entscheidend vom betrachteten Institutionentyp ab. Der Klassifizierung von Institutionen kommt daher für die Betrachtung eine hohe Bedeutung zu. So unterscheidet z.B. Dietl zwischen ,fundamentalen“ und „sekundären“ Institutionen (3). Fundamentale Institutionen wie z.B. das Rechtsempfinden wandeln sich nur langsam und entziehen sich der Gestaltung durch Akteure. Sekundäre Institutionen wie z.B. Gesetze sind dagegen einer Gestaltung zugänglich. Kiwit/Voigt unterscheiden sogar fünf Kategorien von Institutionen (4): Vier Typen von internen, d.h. nicht staatlich durchgesetzten, Institutionen (Konventionen, ethische Normen, Sitten, formelle private Regeln) und ein externer, d.h. staatlich durchgesetzter, Typ (positives Recht), die sich in der Art der Überwachung unterscheiden. Die unterschiedlichen Institutionentypen erfordern den Rückgriff auf jeweils andere Disziplinen zu ihrer Erklärung: Konventionen, Normen, Sitten erschließen sich häufig erst durch soziologische und anthropologische Untersuchungen, positives Recht bedarf der juristischen und historischen Untersuchung. Ökonomische Designprinzipien ziehen sich quer durch die Institutionengestaltung. Interessanterweise zeigt sich, daß Institutionen dabei heute in Theorieansätzen mehrerer gesellschaftswissenschaftlicher Disziplinen als ein rekursives Phänomen wahrgenommen werden: Institutionen gehen als Rahmenbedingungen in das Handeln von Akteuren ein, gleichzeitig sind sie aber auch Ergebnis eben dieses Handelns. Institutionengestaltung kann als Spiel aufgefaßt werden, in dem die Spieler die Regeln des Spiels selbst mit weiterentwickeln (5). Entsprechende Interpretationen finden sich in der Neuen Institutionenökonomik ebenso wie in der Policy-Analyse oder in soziologischen Strukturtheorien wie derjenigen von Anthony Giddens (6).

2. Durch eine Perspektive, die Institutionen als Bedingung und als Ergebnis des Handelns von Akteuren sieht, stellt sich die Frage, wer denn eigentlich die Akteure sind, die an der (Re-)Produktion von Institutionen mitwirken. Herkömmliche politische Theorie und klassische wirtschaftspolitische Modelle geben hierauf in der Regel eine einfache Antwort: Der Staat ist der zentrale Institutionengestalter. In einer Zeit, in der immer mehr gesellschaftlicher Regelungsbedarf auf internationaler Ebene entsteht, sich im nationalen Raum die Steuerungsgrenzen von staatlichem Handeln zeigen und durch eine große Vielfalt von Steuerungsmodellen abgelöst werden, lassen sich diese Vorstellungen nicht mehr halten. Es bedarf ausdifferenzierterer Steuerungsmodelle, die Anleihen aus anderen Theoriegebieten wie z.B. der Systemtheorie machen.

3. Je stärker Institutionengestaltung als ein polyzentrisches Projekt wahrgenommen wird, desto drängender werden normative Fragen. Denn solange ein demokratischer Staat Institutionen (vermeintlich) vorgibt, ist die (demokratische) Legitimation dieses Handelns geklärt. Wenn Unternehmen, Bürgergruppen, Medien und andere Akteure das institutionelle Design einer Gesellschaft mitgestalten, stellt sich die Frage nach der Legitimation dieses Handelns neu. Für die normativen Grundlagen einer solchen ,Netzwerkgesellschaft" liegen heute erste prozessurale Vorschläge vor (7).

\section{Wege zu institutionellen Reformen}

Gerade für die Politikberatung ist die interdisziplinäre Analyse der heutigen Institutionenlandschaft aber nur ein erster Schritt zu konkreten Impulsen einer institutionellen Umgestaltung:

- Wie „neue“ Institutionen konkret auszusehen haben, läßt sich nicht theoretisch entwickeln und entscheiden. Dazu bedarf es vielmehr lebensweltlich inspirierter Kompetenz und Kreativität, die konkete Institutionenideen im jeweiligen sozialen Kontext entwickelt. Ideen wie Spendenparlamente, Nachhaltigkeitslotterien oder Bürgerforen für Unternehmen (8) entstehen bei Akteuren vor Ort. Institutionenforschung und beratung muß sich daher - ganz im Sinne der Transdisziplinaritätspostulate - um die Einbeziehung solcher lebensweltlichen Experten bemühen.

- Politikberatung, die auf Institutionenumgestaltung zielt, trifft zumeist ins „Herz" des (staatlichen) Auftraggebers. Sie bietet nämlich in der Regel keine einfachen Instrumente, die staatliche Akteure lediglich anzuwenden bzw. einzuführen brauchen, sondern stellt die Organisation und Aufgabendefinition der staatlichen Akteure selbst in Frage. Auch dies erfordert einen frühen und engen Einbezug der beratenen Akteure in den Beratungsprozeß

In der Studie „Institutionelle Reformen für eine Politik der Nachhaltigkeit" haben wir versucht, einen entsprechenden transdisziplinären Weg zu

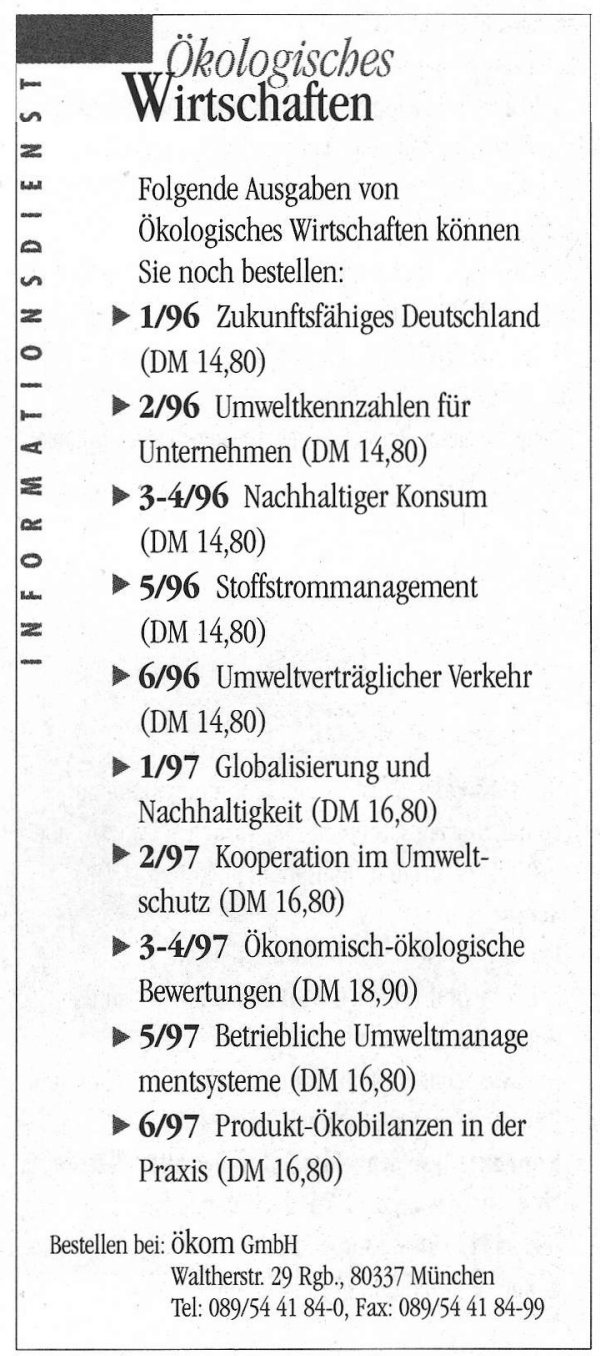


gehen. Dabei zeigte sich, daß die Umsetzung der hier skizzierten Postulate sowohl an Auftragnehmer als auch Auftraggeber einer solchen institutionenbezogenen Politikberatung hohe Anforderungen stellt. Eine transdisziplinäre Institutionenperspektive erwies sich auch in diesem Projekt als ein Forschungs- und Beratungsfeld von hoher praktischer und theoretischer Relevanz, dem es sich auch in Zukunft zu stellen lohnt.

\section{Anmerkungen}

(1) Ein Beispiel bietet die von den Autoren verfaßte Studie "Institutionelle Reformen für eine Politik der Nachhaltigkeit“ für die Enquete-Kommission „Schutz des Menschen und der Umwelt". Die Studie erscheint als: Arbeitsgemeinschaft IWÖ/IFOK: Institutionelle Reformen für eine Politik der Nachhaltigkeit im Frühjahr 1998 im Springer Verlag (Berlin, New York). Eine Zusammenfassung der Studie findet sich in GAIA 6/3 (1997), S.182-197.

(2) Vgl. zur Neuen Institutionenökonomik z.B. R. Richter, E. Furubotn: Neue Institutionenökonomik. Tübingen 1996.

(3) H. Diett: Institutionen und Zeit. Tübingen 1983, S. 71 ff.

(4) D. Kiwit, S. Voigt: Überlegungen zum institutionellen Wandel unter der besonderen Berücksichtigung des Verhältnisses interner und externer Institutionen, in: Ordo, Jg. 46 (1995),

S. 117-147.

(5) Zu einer solchen spielbezogenen Interpretation gesellschaftlicher Zusammenhünge vgl. insbesondere die Analyse von A. Burkard: Markt als Gesellschaftsspiel. Bern 1997 (siehe auch Rezension in diesem Heff).

(6) A. Giddens: Die Konstitution der Gesellschaft. Frankfurt und New York 1988.

(7) Vgl. z.B. D. Messner: Die Netzwerkgesellschaft. Köln 1995.

(8) Vgl. zu diesen Vorschlägen die Enquete-Studie „Institutionelle Reformen" a.a.0.

\section{Die Autoren}

Dr. Hans Peter Meister, Dipl. Pol. Peter Henning Feindi, Jochen Tscheulin, MA, Jürgen Wüst, MA, alle Institut für Organisationskommunikation (IFOK), Bensheim.

Dr. Jürg Minsch, Lic. oec. Tobias Schulz, beide Institut für Wirtschaft und Ökologie an der Universitüt St. Gallen (IW0̈-HSG).

Dr. Uwe Schneidewind, Lehrstuhl für Produktion und Umwelt, Universität Oldenburg.

Kontakt: Uwe Schneidewind, Universitüt Oldenburg, FB 4, Birkenweg 5, D-26111 Oldenburg, Tel: 0441/798-8255, -8300 ,

e-mail: schwind@hrzl.uni-oldenburg.de.

Herausforderungen für eine transdisziplinäre ökologische Wirtschaftslehre

\title{
Zwischen Arbeitsmarkt und wissenschaftlichen Idealen
}

\author{
In den letzten Jahren sind einige ökologische Studienangebote mit inter- \\ oder transdisziplinärem Anspruch in den Wirtschaftswissenschaften \\ entstanden. Die Autoren beleuchten insbesondere vor ihren in St. Gallen und \\ Oldenburg gemachten Erfahrungen, welche Herausforderungen sich solchen \\ Studiengängen stellen und wie diesen zu begegnen ist.
}

$\mathrm{N}$ Von Christoph Schwarzer und Uwe Schneidewind schaften (1) ziehen seit einigen Jahren auch viele wirtschaftswissenschaftliche Fakultäten mit der Einrichtung interdisziplinärer ökologischer Studienangebote nach (2). In einigen Fällen beschränkt sich dies auf einen im wesentlichen betriebs- und volkswirtschaftlichen Kern, in anderen Fällen erstreckt sich das Studienangebot gleichberechtigt auf natur-, wirtschafts- und geisteswissenschaftliche Bezüge wie z.B. beim Studiengang Umweltwissenschaften an der Universität Lüneburg. Von Wissenschaftlern und Praktikern wird zunehmend der Vorwurf laut (3), hier würden Absolventen zur Arbeitslosigkeit ausgebildet, da diese Studiengänge von ,allem ein bißchen, aber nichts richtig" vermittelten. Dies sei gerade angesichts der wieder geringeren Aktualität des Themas "Ökologie“" für die Arbeitsmarktchancen von Absolventen besonders bedenklich.

Dieser Gefahr ist nur dadurch zu begegnen, daß die spezifischen Herausforderungen, vor denen solche interdisziplinären Studiengänge stehen, genau analysiert und die Studienangebote entsprechend darauf ausgerichtet werden.

\section{- Externe und interne Herausforderungen}

Die angespannte Arbeitsmarktlage führt dazu, daß sich in den letzten Jahren die Arbeitsmarktchancen für Volks- und Betriebswirte erheblich verschlechtert haben. Die Studierenden reagieren darauf, indem sie sich in ihren Studienvertiefungen auf klassische (betriebswirtschaftliche) Kernfächer wie z.B. Rechnungswesen/ Controlling, Marketing, Wirtschaftsinformatik konzentrieren, bei denen die Arbeitsmarktchancen noch relativ gut sind. Ein
Studienschwerpunkt "Ökologie" hat deswegen mit der Gefahr zu kämpfen, daß er als karrierehinderlich und arbeitsplatzabträglich angesehen wird.

Viele der heutigen Stellen und Aufgaben im Bereich des Umweltmanagements erscheinen den Studierenden als wenig attraktiv, da sie in vielen Unternehmen immer noch eine Karrieresackgasse bedeuten, d.h. wenige Weiterentwicklungsmöglichkeiten öffnen. Zudem ist die Konkurrenz von Bewerbern aus Nachbardisziplinen (insbesondere aus den Ingenieur- und Naturwissenschaften) sehr hoch.

Aufgrund der Tatsache, daß neben einem ökologischen Schwerpunkt, der einen Großteil des frei wählbaren Hauptstudiumspensums umfaßt (in Oldenburg oder St. Gallen z.B. 24 Semesterwochenstunden), für die Studierenden auch die Möglichkeit besteht, Ökologie lediglich durch kleinere Wahlblöcke oder Einzelveranstaltungen abzudecken, entsteht an den Universitäten eine interne Wettbewerbssituation in der ökologischen Ausbildung: Studierende mit ökologischem Interesse sind nicht darauf angewiesen, den ökologischen Schwerpunkt zu wählen, sie können dieses Interesse auch durch die Belegung anderer - weniger aufwendiger - Veranstaltungen befriedigen. Angesichts der oben beschriebenen externen Herausforderungen besteht die Gefahr, daß sich viele Studierende für eine solche Variante entscheiden.

Das Studienangebot im ökologischen Schwerpunkt ist - wie in den meisten heute angebotenen ökologischen Vertiefungen - in erster Linie durch die Angebote der daran interessierten Dozenten geprägt. Es ist deswegen von der Konstruktion angebots-und nicht nachfrageorientiert. Es besteht die Gefahr, daß eine Summe von interessanten Einzelaspekten im ökologischen Schwerpunkt vermittelt wird, eine inhaltliche 
(c) 20I0 Authors; licensee IÖW and oekom verlag. This is an article distributed under the terms of the Creative Commons Attribution Non-Commercial No Derivates License (http://creativecommons.org/licenses/by-nc-nd/3.o/), which permits unrestricted use, distribution, and reproduction in any medium, provided the original work is properly cited. 